Ann. Abeille, rg67, 10 (r), 53-6r.

\title{
ÜBER DEFORMATIONEN DES CHITINPANZERS VON HONIGBIENENKÖNIGINNEN (APIS MELLIFICA L.)
}

\author{
R. BÄHRMANN
}

Lehr- und Forschungsanstalt für Bienenzucht. Bezirk Gera, Allemagne (D. D. R.)

\section{EINLEITUNG UND UNTERSUCHUNGSMETHODIK}

Es kommt hin und wieder vor, dass Bienenköniginnen vor dem Schlïpfen aus der Zelle oder kurz danach zugrunde gehen. Von verschiedenen Imkereibetrieben wurden uns solche toten Königinnen zur Untersuchung ïbersandt. An toten Tieren lassen sich aber leider keine eventuellen Schädigungen der inneren Organe mehr erkennen. Derartige Schädigungen könnten die Ursache für das baldige Absterben der Königinnen sein. Wir mussten uns deshalb auf eine Untersuchung des Chitinpanzers beschränken. Abnorme Veränderungen des Chitinpanzers konnten zumindest bei einem Teil dieser Königinnen erwartet werden, worauf die Befunde an einzelnen Königinnen früherer Untersuchungen hindeuten (BÄHRMANN, I965a). Die damals erzielten Ergebnisse waren der Anlass fïr die vorliegenden umfangreicheren Untersuchungen.

Anomalien treten mitunter im Gefolge von Entwicklungsstörungen auf (FYG, I958; MAAs, I949; MichaILOFF, I927 ; Tischi,ER, I958; WEISS, I962). Entwicklungs störungen haben aber möglicherweise auch hier zum frühzeitigen Absterben der 'Tiere gefiihrt.

Fine interessante Chitinanomalie bei einer Bienenkönigin beschreibt Nelson rgI2.

Die Königinnen wurden zuerst äusserlich auf etwaige Schädigungen mit Hilfe cles Binokulars untersucht. Danach erfolgte eine Trennung des Abdomens vom iibrigen Körper. Das Abdomen wurde in Io p. Ioo iger $\mathrm{KOH}$ erweicht, in $\mathrm{H}_{2} \mathrm{O}_{2}$ aufgehellt und segmentweise auf Schädigungen gepriift, wobei der Stachelapparat besondere Berïcksichtigung fand.

Für die vorliegenden Untersuchungen standen I69 Königinnen zur Verfügung, die in den Jahren I964-I965 gesammelt worden sind. 


\section{UNTERSUCHUNGSERGEBNISSE}

Von den I69 Königinnen liessen 96 Schädigungen am Chitinpanzer erkennen, wobei sich die Anomalien folgendermassen verteilen : bei 40 Tieren im Bereich des Stachelapparates, bei 29 Tieren an den Thorakal- und Abdominal-segmenten, $26 \mathrm{mal}$ Häutungsstörungen, I4mal Flügelanomalien, r3mal unausgebildete Flïge1, 6mal Beinanomalien, 3mal Deformationen der Mundteile and $3 \mathrm{mal}$ Deformationen der Fühler. In einem Falle konnte eine konkave Einwölbung der Stirn über der Fühlerbasis beobachtet werden. Normalerweise tritt die Stirn leicht konvex hervor. Verschiedentlich treten Deformationen mehrerer Körperteile an einunddemselben Tier auf, ohne dass jedoch eine bestimmte Beziehung zwischen den Anomalien der geschädigten Körperteile zu erkennen wäre.

Die Deformationen der Fïhler bestehen im Fehlen ganzer Teile der Geissel. Die übrig gebliebenen Fühlerstümpfe geben einen Hinweis dafür, dass die Geisselglieder nicht post mortal abgebrochen sein können. Die anomalen Rïssel sind rudimentär und abnorm gekrümmt. Beinanomalien treten vor allem am 2. und 3 . Beinpaar auf. In den 6 vorliegenden Fällen sind entweder rechts oder links oder beiderseits nur Extremitätenstümpfe zu beobachten.

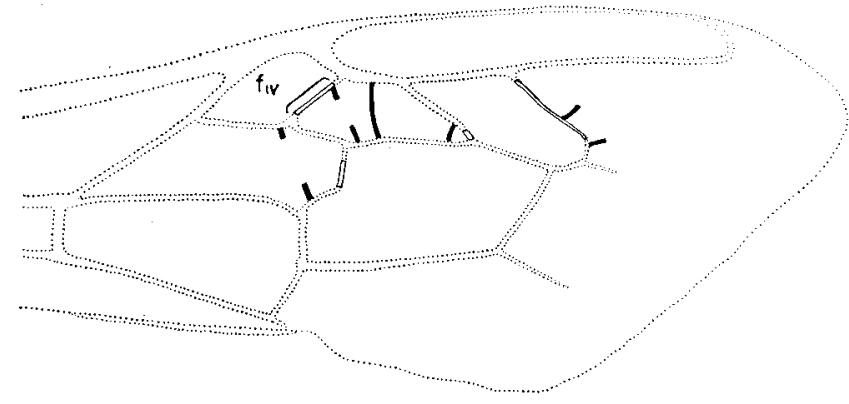

Aвв. I. - Rechier Vorderflügel einer Königin

In den Flügel sind sämtliche bei den r69 untersuchten Königinnen aufgefundenen Aderanomalien eingetragen; $\boldsymbol{\square}$ : zusätzliche Aderstrecken; $\bullet$ : fehlende Aderstrecken; weitere Erläuterungen im Text.

Fig. I. - Aile avant droite d'une reine

(toutes les anomalies véneuses trouvées chez les 169 reines observées ont été indiquées sur l'aile) - nervures supplémentaires $[.7$ nervures manquantes. Pour plus d'explications voir texte

Flügelanomalien kommen in zwei Formen zum Ausdruck : entweder sind die Flügel überhaupt nicht oder kaum entwickelt, wobei lediglich kleine weissliche Stummel ohne jede Andeutung einer Aderung vorhanden sind, oder die Fliugel sind zwar in normaler Grösse ausgebildet, das Flügelgeäder ist jedoch anomal. Bei 4 der I4 Königinnen mit Fliigeladeranomalien sind zwei oder mehr Anomalien ausgebildet. Bemerkenswert ist, dass Aderanomalien nur an den Vorderfligeln auftreten, im Gegensatz zu Arbeitsbienen und Drohnen (BÄHrmann, I963). Eine der Anomalien (Abb. I, $f_{\mathrm{vI}}$ ) konnte bei den bisher untersuchten II 226 Arbeitsbienen und Drohnen nocht nicht festgestellt werden, obgleich bei ihnen bis jetzt 92 unterschiedliche Aderanomalien zu beobachten waren (B̈̈HRMANs, im Druck). Die Anomalien sind 


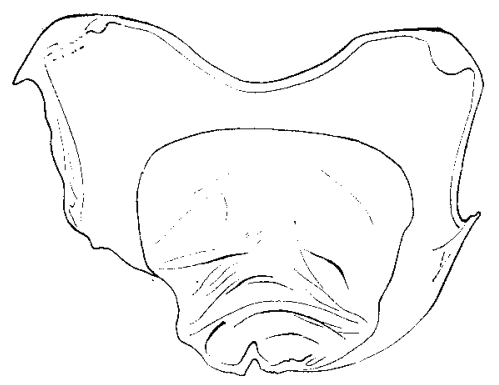

Aвв. 2. - $\gamma_{\mathrm{e}}$ Abdominalsternit einer Königin

(das Sternit ist asymmetrisch und zeichnet sich durch Querfaltungen aus)

FIG.2. - $7^{\circ}$ slernite abdominal d'une reine

(le sternite est asymétrique et se distingue par des plis transversaux)
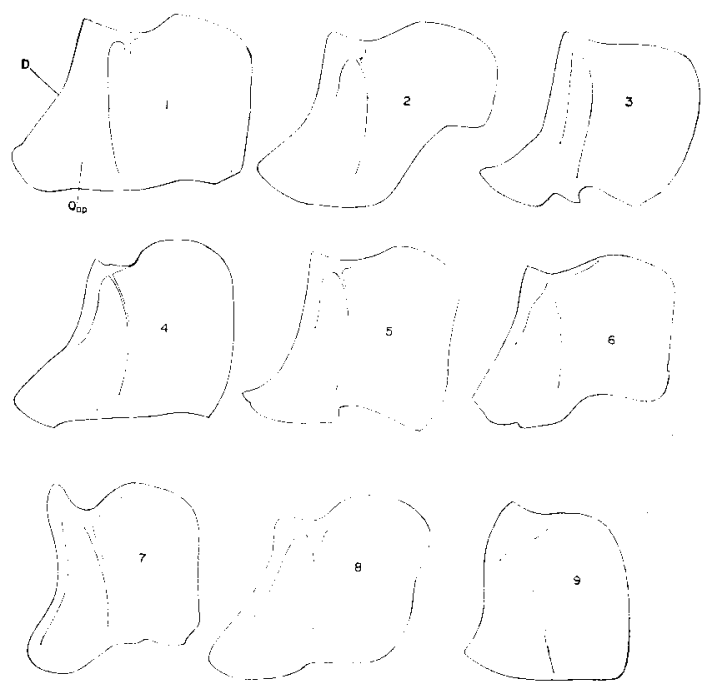

Авв. 3. - Verschiedene Formen quadratischer Platlen des Stachelapparates gesunder Königinnen D, Dorsalkante der quadratischen Platte; Qap, apodemaler Anteil der quadratischen Platte

FIG. 3. - Différentes formes de plaques angulaires de l'appareil vulnérant d'une reine saine $\mathrm{D}$, arête dorsale de la plaque angulaire; Qap, apodème de la plaque angulaire 
entweder symmetrisch an beiden Vorderflïgeln oder auch nur einseitig links bzw. rechts vorhanden.

An einer grösseren Anzahl von Königinnen (26) waren Häutungsreste zu erkennen, die sich an verschiedenen Körperteilen befanden. Es sei darauf hingewiesen, dass es sich dabei meist um solche Königinnen handelt, die vor dem Schlïpfen in der Zelle abgestorben sind. Häutungsreste sind hauptsächlich am 3., seltener am 2. Beinpaar, an den unausgebildeten Flïgeln, dem Kopf und am Hinterleibsende sichtbar. Mit Ausnahme der Flïgel lassen sich keine Beziehungen zwischen den Häutungsresten und den geschädigten Beinen oder Abdominalsegmenten nachweisen.

Die Segmentanomalien erstrecken sich zumeist auf die Abdominalsegmente. Sie bestehen in Faltenbildungen, konkaven Einwölbungen der normalerweise konvex gewölbten Segmentteile und in Asymmetrien. Faltenbildungen treten vor allem am 7. Abdominalsegment auf (Abb. 2). Die Tergite bzw. Sternite erhalten wohl dadurch sekundär eine mehr oder weniger asymmetrische Form. Verschiedentlich kommen an den Sterniten, aber manchmal auch an den Tergiten Foramina vor, wie sie bereits beschrieben worden sind (B̈̈нRMANx, I965 a). Die Deformationen an den Thorakalsterniten beziehen sich jeweils auf abnorme seitlich gelegene Einbuchtungen.

Der Stachelapparat der Königinnen zeigt die häufigsten abnormen Veränderungen, die sich auf alle chitinigen Bestandteile desselben beziehen können. Um klare Aussagen über anomale Bildungen des Stachelapparates vornehmen zu können, ist es erforderlich, seine Bestandteile in normalen Zustand genau zu kennen. Deshalb wurden an gesunden Königinnen Untersuchungen des Stachelapparates vorgenommen, die zu dem Ergebnis geführt haben, dass die chitinigen Stachelteile, vor allem die quadratischen Platten und die Stigmenplatten, in weit höherem Masse Formverschiedenheiten zeigen als dieselben Teile des Stachelapparates der Arbeitsbienen. So ist z. B. die Variabilität der quadratischen Platten recht gross (Abb. 3). Die Dorsalkante (D) ist mehr oder weniger geschwungen und kann distal in einen
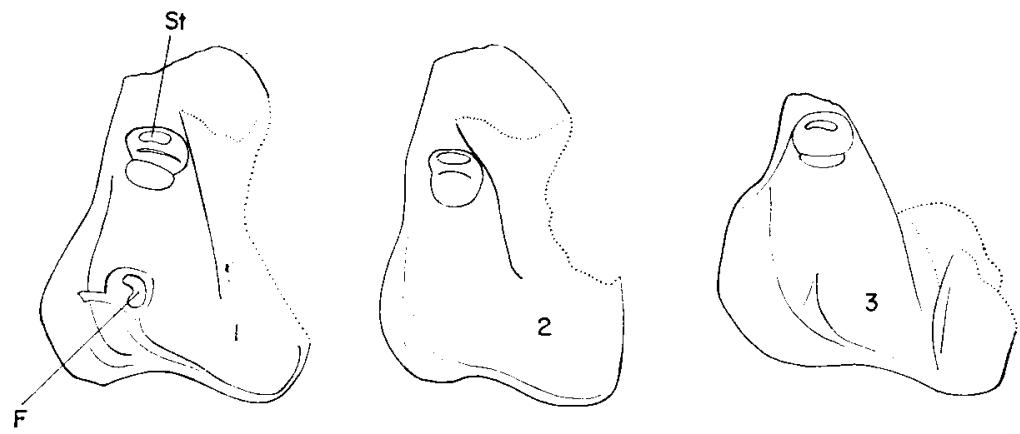

Авв. 4. - Drei unterschiedliche Typen von Stigmenplatten gesunder Königinnen

F, Formmen; St, Stigma

FiG. 4. - 3 types différents de plaques slignatiques de reines saines

F, Foranen ; St, Stignate

Zipfel auslaufen $(3,5)$. Der apodemale Anteil der Platten, in einigen Fällen mit dem übrigen Plattenteil nur lose verbunden, ist sehr gross (4), kann aber auch häufig unbedeutend sein (9). Ähnliche Verhältnisse liegen bei den Stigmenplatten vor. Drei häufige Typen sind in Abbildung 4 dargestellt. Der Stigmenplatten-Index beträgt 
im Durchschnitt I.83 und liegt damit selbst iiber den höchsten mittleren Indexwerten, die bei Arbeitsbienen der Subspecies Apis m. mellifica ermittelt werden konnten (r.65, Herkunft : Thüringen, D.D.R. ; I.66, Herkunft : Baschkirien, Sowjet-Union) (BÄHRMANN, I 965 b). Trotz der grossen Variabilität dieser Plattenteile des Stachelapparates liess sich bezüglich der rechten und linken Plattenseite Kollektivsymmetrie ermitteln. Die Lage des Stigmas ist unterschiedlich. Manchmal endet die Stigmenplatte direkt über dem Stigma (3). Von dieser Form bis zu deutlich abnormen Stigmenplatten gibt es eine Reihe von Übergängen, so dass es nicht immer möglich ist, eine normal geformte von einer deformierten Stigmenplatte zu unterscheiden. Deformationen werden deutlich, wenn die Dorsalkante der Platten umgebogen ist oder wenn sich im proximalen Plattenteil Foramina befinden. Ieutlich abnorme Veränderungen kommen in Abbildung $5 a$ an einer quadratischen Platte zum Ausdruck. Die abnorme Form der quadratischen Platte hängt wohl mit den Deformationen der iibrigen Stachelteile zusammen, von denen auf der Zeichnung nur die ebenfalls deformierte oblonge Platte dargestellt ist. Die häufigen Anomalien des Stachels sind sicherlich auf die mangelhafte Verbindung zwischen Stechborsten und Stachelrinne zurïckzuführen, die einen unterschiedlichen Ausbildungsgrad erreichen kann (Abb. $6 a, b)$. Die Abbildung $6 c / d$ veranschaulicht einen Stachel, dessen Stachelrinnenkolben deformiert ist. Dasselbe gilt vom Basalteil der Stechborsten $(d)$, besonders auffällig ist dabei median die breite spaltförmige Öffnung zwischen den Stechborsten. Ausserdem sei auch auf die geringe Krïmmung des Stachels hingewiesen, eine Anomalie die sehr häufig ist.

Aus einer Übersicht über die beobachteten Anomalien an den einzelnen Teilen des Stachelapparates ('Tab. I) geht hervor, dass die Stechborsten und die Stache1rinne am häufigsten I)eformationen aufweisen. In einem Falle waren die Stechborsten iiberhaupt nicht ausgebildet. An diesem Stachelapparat fehlte auch die Furcula (Nr. I). Bei Deformationen der Furcula handelt es sich im übrigen um Asymmetrien der Gabelung. In Tabelle I kommt weiterhin zum Ausdruck, dass Deformationen an den Stachelteilen meistens symmetrisch auftreten. Bei etlichen Tieren sind mehrere Teile des Stachelapparates zugleich deformiert ; besonders ausgeprägt ist das bei den Nummern $I, 2,9$ und $I 3$.

\section{DISKUSSION DER ERGEBNISSE}

Es ist naheliegend, die Deformationen des Chitinpanzers der Königinnen in Zusammenhang mit dem baldigen Absterben der Tiere zu bringen. $\mathrm{Ob}$ diese Deformationen dafïr allerdings den einzigen Grund darstellen, lässt sich nicht sagen. Handelt es sich bei den abnormen Veränderungen des Chitinskeletts um Folgen von Entwicklungsstörungen, ist anzunehmen, dass ausser der Körperhülle auch noch andere Organe oder deren Teile geschädigt worden sind. Das dürfte bei Anomalien an den Körpersegmenten oder am Stachelapparat z. B. für die dort inserierenden Muskelbündel zutreffen. Welche der hier beschriebenen Anomalien allein auf Entwicklungsstörungen beruhen und welche eventuell erblich bedingt sind, ist nicht ohne weiteres zu klären. Es wäre wïnschenswert, in entsprechenden experimentellen Untersuchungen aufzuklären, welche der hier beobachteten Schädigungen zustande 


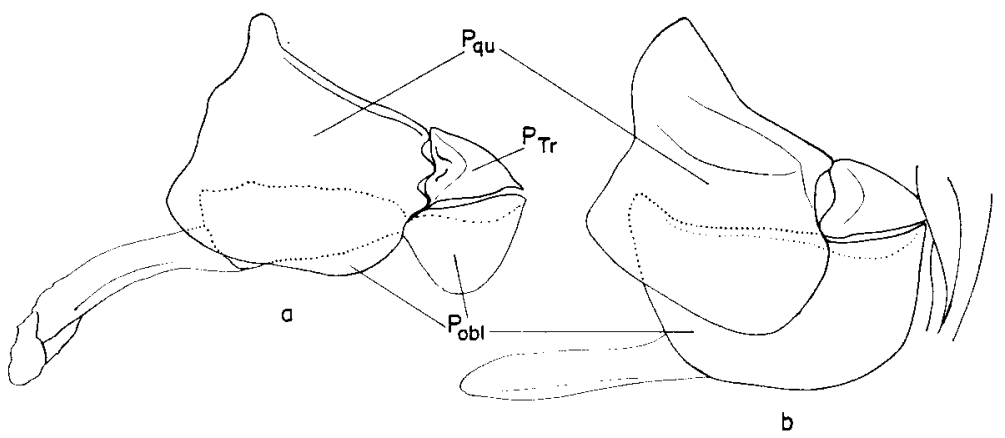

Aвв. 5. - Seitenansicht des Slachelapparates zweier Königinnen

a) deformiert, b) normal ausgebildet; $\mathrm{P}_{\mathrm{obl}}$, oblonge Platte; $\mathrm{P}_{\mathrm{gu}}$, quadratische Platte; $\mathrm{P}_{\mathrm{Tr}}$, Triangularplatte

FIG. 5. -- Vue latérale de l'appareil vulnérant de 2 reines

a) déformé, $b$ ) normalement constitué ; $\mathrm{P}_{\mathbf{o b l}}$, plaque oblongue ; $\mathrm{P}_{\mathrm{qu}}$, plaque quadrilat.; $\mathrm{P}_{\mathrm{tr}}$, plaque triangulaire

a

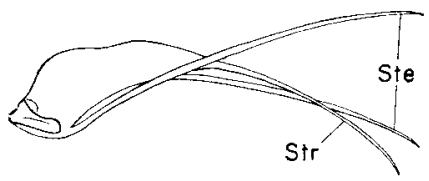

b

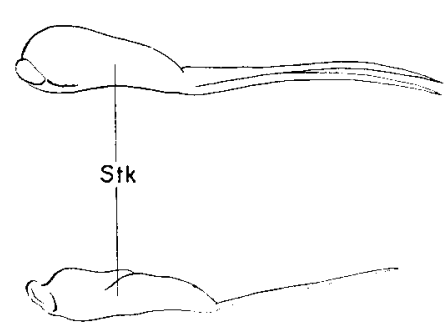

C

d

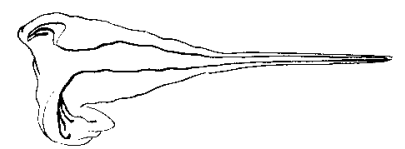

Авв. 6. - Deformierte Stachel von Königinnen;

a) Stechborsten (Ste) und Stachelrinne (Str) sind nicht miteinander verbunden ; b) der Stachel ist kaum gekrümmt, Stechborsten und Stachelrinne sind nur basal miteinander verbunden ; $c$ ) Stachel mit deformiertem Stachelkolben (Stk) und kaum gebogenem Stachel ; $d$ ) derselbe Stachel wie in $c$ ), jedoch hier die Ventralansicht, weitere Ërläuterungen im Text.

FIC. 6. - Aiguillon déformé des reines

a) les soies (Ste) et les gaines de l'aiguillon (Str) ne sont pas liées; $b$ ) l'aiguillon est à peine recourbé, les soies et les gaines ne sont liées qu'à leur base. c) Aiguillon avec un bulbe déformé (Stk) et à peine recourbé, $d$ ) même aiguillon qu'en $c$ ) mais en vue ventrale. Pour plus d'explications voir texte. 


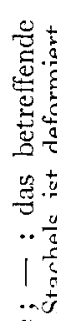

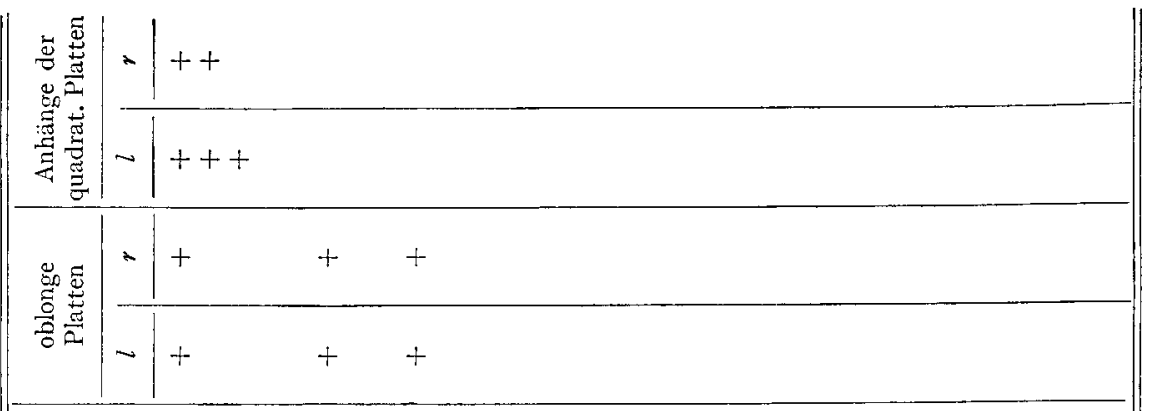

\begin{tabular}{|c|c|c|}
\hline 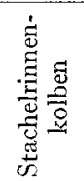 & + & ++ \\
\hline 莺 & $1++$ & + \\
\hline
\end{tabular}

\begin{tabular}{|c|c|c|c|c|c|}
\hline \multirow{2}{*}{ 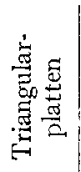 } & a & ++ & + & + & + \\
\hline & & $+t$ & + & t & + \\
\hline
\end{tabular}

\begin{tabular}{|c|c|c|c|c|c|c|}
\hline : & 1 & + & + & + & + & + \\
\hline 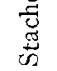 & $\sim$ & + & + & + & & + \\
\hline
\end{tabular}

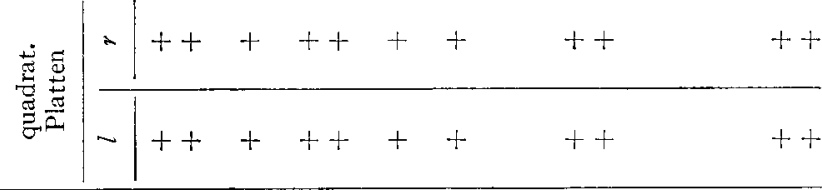

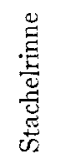

$$
++++\quad+++++
$$

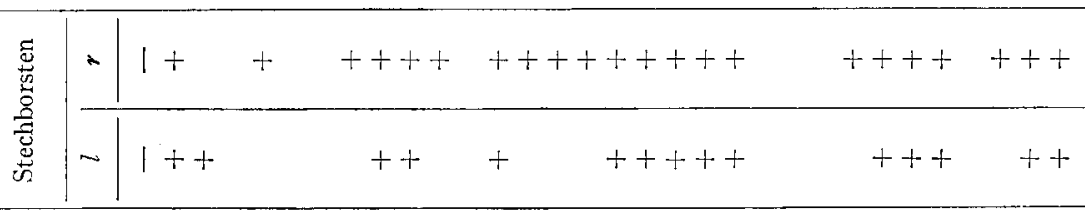


kommen, wenn keine normalen Entwicklungsbedingungen herrschen. Die realtiv häufigen Deformationen an den Körperanhängen, vor allem an den Flügeln, und die Anomalien am Ende des Abdomens (die letzten Tergite und der Stachelapparat) sprechen für Entwicklungsstörungen ; dabei ist jedoch die Möglichkeit nicht ausgeschlossen, dass bei einzelnen Tieren auch eine durch Mutation verursachte Flïgelreduktion (KERR und LAIDLAW, I956) vorliegen könnte. Auch Häutungsstörungen könnten möglicherweise in einzelnen Fällen für Deformationen des Chitinpanzers verantwortlich sein. FYG (I958) fand in Folge von Häutungsstörungen Verkrüppelungen der Fühler, Mundteile und Beine bei Arbeitsbienen und Drohnen. Häutungsanomalien könnten natürlich andererseits auch Begleiterscheinungen von Entwicklungsstörungen an einzelnen Körperteilen sein (Körperanhänge, Abdomen), die durch äussere Einflüsse zustande kommen. Solche Einflüsse können in nicht optimalen Brutnesttemperaturen oder dem zu früh erfolgten Verschulen der Weiselzellen durch den Imker bestehen.

$$
\text { Reçu pour publication en décembre Ig66. }
$$

\title{
RÉSUMÉ
}

\author{
SUR LES DÉFORMATIONS DE LA CUIRASSE CHITINEUSE \\ DES REINES D'ABEILLE " APIS MELLIFICA "L.
}

Sur 169 reines mortes avant ou peu après l'éclosion, on a recherché les imperfections de la cuirasse chitineuse. On a considéré différentes parties du corps : tête, thorax, aile, extrémités, segments abdominaux et appareil vulnérant. 96 reines avaient des malformations plus ou moins évidentes d'une partie quelconque de leur corps. On a observé assez couramment des imperfections de l'appareil à venin, des tergites abdominaux et des ailes. On suppose qu'il faut attribuer en premier lieu la responsabilité de ces imperfections à des dérangements dans le processus du développement.

\section{SUMMARY}

ON DEFORMATIONS OF THE CHITINOUS ELYTRA OF QUEEN BEES "APIS MELLIFICA

On examination, imperfections were found in the chitinous elytra of I 69 queen bees which died before or just after hatching. Different parts of the body were studied : head, thorax, wing, extremities, abdominal segments and stinging apparatus. Malformations of some part of the body of 96 queens were evident to a greater or lesser degree. Imperfections of the venom apparatus, the abdominal tergites, and the wings were quite readily observed. It was concluded that these imperfections must arise in the development process.

\section{ZUSAMMENFASSUNG}

I69 vor oder kurze Zeit nach dem Schlüpfen abgestorbene Königinnen wurden auf Schädigungen des Chitinpanzers untersucht. Dabei fanden folgende Körperteile Berücksichtigung : Kopf, Thorax, Flügel, Extremitäten, Abdominalsegmente und Stachelapparat. 96 Königinnen zeigten mehr oder weniger deutliche Deformationen einzelner Körperteile. Relativ häufig konnten Schädigungen am Stachelapparat, den Abdominaltergiten und den Flügeln beobachtet werden. Es ist anzunehmen, dass für diese Schädigungen in erster Linie Entwicklungsstörungen verantwortlich gemacht werden müssen. 


\section{LITERATUR}

B.hrмaxi R., I963. Zum Vorkommen von Anomalien im Fï̈gelgeäder der Honigbiene. Arch. Bienenkde, 40, $49-58$.

BÄrnmany R., I965a. Durch verschiedene Ersachen bedingte Anomalien bei Honigbienenkoniginnen. Arch. Geflïgelsucht $u$. Kleintierkile, 14, $177^{-189}$.

BÄHRMAns R., I 965 b. Merkmalsstudien und infraspezifische Verwandtschaftsverhailtnisse bei $A p i s$ mellifica L. 7.. Bienentorsch., 8, 17-47.

BäIrknavs R., Ig65 c. Im Druck. Untersuchungen zum Vorkommen von Aderanomalien im Flügelgeäder der IIoniybiene.

FYG W., 1958. C'ber die normale und abnome Entwicklung der Honigbiene. Schaeiz. Bienenzlg., 81 N. IF., $194-200,3+5-355,387-398$.

KERR W. I., LAIDLAW H. II., I956. (seneral Genetics of Bees. Advances in Genetics, 8, 109-I53.

MaAs A.-IL, 1949. Ujber die Auslüsbarkeit von Temperatumoditikationen während der limbryonalentwickhung von Drosophila melanogaster MEIGEx. Roux. Arch. Entrickl. mech. Organismen, 143, 5I 5-572.

Mrchalof: A. S., I927. Der Finfluss einiger Lebenslagefaktoren auf die Variabilität der Honigbiene. Arch. Bienenkde, 8, 289-303.

Nelson, J. A., 1912. Structural pecularities in an abnormal queen bee. Proc. Acal. nat. Sci. Philadelphia, 64, 3-5.

Tischler W., I958. Beobachtungen über Entwicklungsabnornitäten bei Insekten. Zool. Anzeiger, 160, I I 2-I I 4.

Wenss K., I962. Über die Lebensfähigkeit von offener und gedeckelter Brut ausserhalb des Bienenvolkes. Z. Bienentorsch, 6, 104-II 4 .

Neue Anschrift des Verfassers :

Dr. R. Bährmann, Institut für Spezielle Zoologrie und Entomologie, 69 Jena, Fraunhoferstrasse 6 D. D. R. 